\title{
EFFECT OF FRESH FROZEN PLASMA TRANSFUSION ON PROTHROMBIN TIME IN PATIENTS WITH MILD COAGULATION ABNORMALITIES WHEN COMPARED WITH MAJOR COAGULATION ABNORMALITIES
}

\author{
Sasikala Nadanganan', Mary Sanshya², Meena Dharmadas ${ }^{3}$ \\ ${ }^{1}$ Associate Professor, Department of Transfusion Medicine, Government TDMC, Alappuzha. \\ 2Junior Resident, Department of Transfusion Medicine, Government TDMC, Alappuzha. \\ 3Professor, Department of Transfusion Medicine, Government TDMC, Alappuzha.
}

\section{BACKGROUND}

ABSTRACT

Fresh Frozen Plasma (FFP) is frequently transfused to patients with mild to moderate elevations in Prothrombin time (PT) and International Normalized Ratio (INR) under the twin assumptions that these tests imply a coagulopathy and that FFP transfusion will correct the coagulopathy. This study gives an insight into the pattern and appropriateness of fresh frozen plasma transfusion practice among various clinical departments.

\section{MATERIALS AND METHODS}

This is a prospective cohort study done for a period of 2 years from 2013 January on all FFP transfusions for mild and major coagulation abnormalities. Mild means PT 13.1 to 17 seconds and INR 1.1 to 1.85 , major means, PT more than 17 seconds and INR more than 1.85. In all patients of both the groups' pre-transfusion PT INR values and post transfusion PT INR values 6 to 8 hours after FFP transfusion were assessed.

\section{RESULTS}

In this study among the 60 major abnormality cases, $86.7 \%$ showed significant improvement in PT INR following FFP transfusion ( $p<0.001)$, whereas in 60 mild cases, only $5 \%$ had significant improvement $(p=0.55)$.

\section{CONCLUSION}

The study revealed that, there was significant improvement in PT INR values after FFP transfusion in major coagulation abnormalities. In mild elevation of PT INR, FFP transfusion did not show any improvement. The study also showed that there is a widespread uncertainty about the appropriate use of FFP among our clinicians despite the existence of various guidelines, resulting in high rate of inappropriate transfusions.

\section{KEYWORDS}

Fresh Frozen Plasma, Prothrombin Time, International Normalized Ratio, Coagulopathy.

HOW TO CITE THIS ARTICLE: Nadanganan S, Sanshya M, Dharmadas M. Effect of fresh frozen plasma transfusion on prothrombin time in patients with mild coagulation abnormalities when compared with major coagulation abnormalities. J. Evolution Med. Dent. Sci. 2018;7(08):1002-1007, DOI: $10.14260 /$ jemds/2018/229

\section{BACKGROUND}

Fresh frozen plasma is a blood product extracted from plasma and frozen to $-18^{0} \mathrm{c}$ or below within six hours after collection. It contains plasma proteins and all the coagulation factors including the labile factors V and VIII. Fresh Frozen Plasma is frequently transfused to patients with mild to moderate elevations in Prothrombin Time (PT) under the twin assumptions that these tests imply a coagulopathy and that FFP transfusion will correct the coagulopathy.

The appropriate use of FFP requires an understanding of the properties of FFP and its inadequacies, as well as an appreciation of its complications. In spite of clear guideline regarding the use of FFP, many studies have shown a high incidence of inappropriate use of FFP. ${ }^{1}$

'Financial or Other Competing Interest': None.

Submission 24-11-2017, Peer Review 05-02-2018,

Acceptance 12-02-2018, Published 19-02-2018.

Corresponding Author:

Dr. Sasikala N,

Associate Professor

Department of Transfusion Medicine,

Government Medical College,

Thiruvananthapuram.

E-mail: dr.sasikala.n@gmail.com

DOI: $10.14260 /$ jemds/2018/229
The College of American Pathologists (CAP) and the British Committee for standards in Haematology (BCSH) have published guidelines to highlight these issues and minimize misuse Literature search revealed only few audits from India. ${ }^{2-4}$ Many studies have shown a high incidence of inappropriate use of FFP, a policy of mandatory pretransfusion approval, FFP is the blood component most denied or changed.5-8

Inappropriate transfusion therapy with FFP is probably one of the main avoidable risks for patients and it is known that guidelines are not, per se able to influence clinical practice because without the help of other instruments, such as auditing and educational interventions, they may not be able to reach a level of diffusion and acceptance such as to be able to direct clinical practice towards the recommended behaviours. Mild Coagulation Abnormality means Prothrombin time (PT) value between 13.1 to 17 seconds \& International Normalized ratio (INR) value between 1.1 to 1.85. INR is a measure of clotting used to monitor vitamin $\mathrm{K}$ antagonists such as warfarin, and the test has only been validated for use in this clinical setting.

However, it is widely used as the main reporting measure in hospitals - for example, in the Intensive Care Study of Coagulopathy (ISOC) around half of the recruiting centers reported coagulation results in critical care as INRs. Also INR 
is used as a measure of clotting in other settings such as liver disease but again, there are uncertainties whether the INR correlates with bleeding risk in this setting.

The use of FFP has significantly increased in the past 10 years. There are certain situations where FFP is clearly indicated such as coagulation factor deficiency secondary to liver disease, DIC, dilutional coagulopathy due to massive blood transfusion, in infants with secondary immunodeficiency, antithrombin deficiency and open-heart surgery. ${ }^{1}$ Despite the fact that coagulation test variables predict the risk of bleeding has been challenged, the use of FFP before invasive procedures for mildly prolonged PT values is a wide spread practice. The persistence of this transfusion practice may relate to the assumption that FFP corrects the abnormal coagulation results.

FFP contains antibodies against $\mathrm{ABO}$ antigens and is capable of causing complications like haemolytic transfusion reactions and transfusion related acute lung injury. It is also capable of transmitting transfusion transmissible infections. Other complications like allergic reaction and fluid overload associated with blood transfusion can also occur with plasma infusion. Hence, the use of FFP is not without potential damage. In certain situations, like specific factor or fibrinogen deficiency, FFP is not indicated. ${ }^{2}$

Our institute is a tertiary care teaching hospital. Consistent with the global trend, FFP usage has been increasing in our institute since past few years. This study is mainly intended to find out the unnecessary transfusions of FFP and the effect of FFP on coagulation profile by comparing the effect of FFP transfusion on PT INR in mild and major coagulation abnormality cases. We believe, this may help in anticipating the dose of FFP required to improve the coagulation variables and may also help us in setting up policies to improve the utility and reduce the wastage of this important blood product

\section{MATERIALS AND METHODS}

The present study was a prospective cohort study done for a period of 2 years from January 2013. The pre-transfusion PT INR was categorized into 2 groups, mild and major. Mild means PT 13.1 to 17 seconds and INR 1.1 to 1.85 , major means, PT more than 17 seconds and INR more than 1.85 .

Pre-transfusion samples from patients who are included in the study were collected.

$1.8 \mathrm{ml}$ of venous blood was drawn into sodium citrate vacutainer and the PT INR was calculated. FFP transfusion as per request was given to the patient. Post transfusion sample within 6 to 8 hours were taken for PT INR determination. PT was determined with a standard method of determination of the time necessary to coagulate $0.2 \mathrm{ml}$ of plasma at $37^{\circ} \mathrm{C}$ after addition of $0.2 \mathrm{ml}$ of thromboplastin which has an ISI of 2.0 . The whole procedure was done in a Semicoagulometer. The INR was calculated by raising the PT ratio to the power of the recipient ISI. The pre-transfusion and post transfusion PT INR values were recorded for both mild and major coagulation abnormalities.

The change in INR in each case after FFP transfusion was determined and compared to assess their added effect on clinical improvement of the patients. Finally, all suspected transfusion reactions due to FFP or other blood products transfused in conjunction with FFP were also recorded and analysed.

\section{Statistical Analysis}

Statistical data was analysed using SPSS software version 17. Quantitative variables were described by mean, median, standard deviation; minimum, maximum and qualitative variables were described by percentage distribution. Between group comparison of qualitative variables were analysed by chi square test and Pre-test, post-test comparison of quantitative variables were analysed by paired t test.

All values of $\mathrm{p}<0.05$ was considered statistically significant.

\section{RESULTS}

This is a prospective cohort study conducted to compare the Effect of Fresh Frozen Plasma transfusion in mild and major coagulation abnormalities in patients at Government $\mathrm{T}$ D Medical College Alappuzha over a period of 2 years. In our study we received 120 requests for FFP transfusions with increased PT INR values. According to the criteria implied in our study 60 showed mild coagulation abnormality and 60 showed major prolongation.

The guidelines published by CAP, National Health and Medical Research Council (NHMRC) and Australasian Society for Blood Transfusion (ASBT) were used as standards. 3,5

\begin{tabular}{|c|c|c|c|c|}
\hline \multirow{2}{*}{$\begin{array}{c}\text { Indication for FFP } \\
\text { Transfusion }\end{array}$} & \multicolumn{2}{|c|}{$\begin{array}{c}\text { Mild } \\
\text { Coagulopathy }\end{array}$} & \multicolumn{2}{|c|}{$\begin{array}{c}\text { Major } \\
\text { Coagulopathy }\end{array}$} \\
\hline & $\mathbf{N}$ & $\%$ & $\mathbf{N}$ & $\%$ \\
\hline Bleeding & 0 & 0 & 31 & 51.7 \\
\hline Coagulopathy & 0 & 0 & 16 & 26.7 \\
\hline $\begin{array}{l}\text { Pre-operative } \\
\text { prophylaxis }\end{array}$ & 15 & 25 & 9 & 15 \\
\hline $\begin{array}{l}\text { Postoperative } \\
\text { prophylaxis }\end{array}$ & 3 & 5 & 4 & 6.7 \\
\hline $\begin{array}{c}\text { Prophylaxis against } \\
\text { bleeding }\end{array}$ & 42 & 70 & 0 & 0 \\
\hline Total & 60 & 100 & 60 & 100 \\
\hline
\end{tabular}

In patients with mild prolongation of PT INR, $100 \%$ of transfusions were prophylactic whereas in major abnormality, $51.7 \%$ of transfusions were therapeutic for bleeding, and the rest were prophylactic.

\begin{tabular}{|c|c|c|c|c|}
\hline \multirow{2}{*}{ No. of FFP } & \multicolumn{2}{|c|}{ Mild Coagulopathy } & \multicolumn{2}{c|}{ Major Coagulopathy } \\
\cline { 2 - 5 } & $\mathrm{N}$ & $\%$ & $\mathrm{~N}$ & $\%$ \\
\hline $0-1$ & 0 & 0 & 6 & 10 \\
\hline $2-4$ & 59 & 98.3 & 36 & 60 \\
\hline $5-8$ & 1 & 1.7 & 18 & 30 \\
\hline Total & $\mathbf{6 0}$ & $\mathbf{1 0 0}$ & $\mathbf{6 0}$ & $\mathbf{1 0 0}$ \\
\hline Table 2. Distribution of range of FFP transfused in both \\
mild and major coagulopathy \\
\hline
\end{tabular}

The average amount of FFP transfused in both the groups are in the range of 2- 4 units and the median is 2 units. 


\begin{tabular}{|c|c|c|c|c|}
\hline $\begin{array}{c}\text { Pretransfusi } \\
\text { on } \\
\text { PT /INR }\end{array}$ & $\mathbf{N}$ & $\mathbf{\%}$ & $\mathbf{N}$ & $\%$ \\
\cline { 2 - 5 } Prolonged & 0 & 0 & 18 & 30 \\
\hline $51-60 "$ & 0 & 0 & 1 & 1.7 \\
\hline $41-50.9 "$ & 0 & 0 & 3 & 5 \\
\hline $31-40.9 "$ & 0 & 0 & 8 & 13.3 \\
\hline $21-30.9 "$ & 0 & 0 & 17 & 28.3 \\
\hline $17.1-20.9 "$ & 0 & 0 & 13 & 21.7 \\
\hline $11-16 "$ & 48 & 80 & 0 & 0 \\
\hline $16.1-18$ " & 12 & 20 & 0 & 0 \\
\hline Total & 60 & 100 & 60 & 100 \\
\hline Table 3. Distribution of pre-transfusion PT INR in both \\
mild and major coagulopathy \\
\hline
\end{tabular}

Around $80 \%$ of mild coagulation values were in the range of $11-16$ seconds and rest $20 \%$ were in the range of 16.1 18 seconds. $62 \%$ of major coagulation values were in the range of $17.1-40.9$ seconds, and $30 \%$ were giving values as prolonged.

\begin{tabular}{|c|c|c|c|c|}
\hline \multirow{2}{*}{$\begin{array}{c}\text { Post } \\
\text { transfusion } \\
\text { PT/INR }\end{array}$} & \multicolumn{2}{|c|}{ Minor Coagulopathy } & \multicolumn{2}{c|}{ Major Coagulopathy } \\
\cline { 2 - 5 } & $\mathrm{N}$ & $\%$ & $\mathrm{~N}$ & $\%$ \\
\hline $12-14.9$ & 0 & 0 & 9 & 15 \\
\hline $15-17.9^{\prime \prime}$ & 50 & 83.3 & 25 & 41.7 \\
\hline $18-20.9 "$ & 10 & 16.7 & 13 & 21.7 \\
\hline $21-23.9^{\prime \prime}$ & 0 & 0 & 7 & 11.7 \\
\hline $30-32^{\prime \prime}$ & 0 & 0 & 6 & 10 \\
\hline Total & 60 & 100 & 60 & 100 \\
\hline Table 4. Distribution of post transfusion PT /INR range in \\
both mild and major coagulopathy \\
\hline
\end{tabular}

In mild coagulopathy, all cases remained in the same range as their pre-transfusion values, whereas in major 78.4 $\%$ cases showed a decrease in PT value below 20.9.

\begin{tabular}{|c|c|c|c|c|}
\hline \multirow{2}{*}{ PT/INR } & \multicolumn{2}{|c|}{ Mil coagulopathy } & \multicolumn{2}{c|}{ Major coagulopathy } \\
\cline { 2 - 5 } & $\mathbf{N}$ & $\mathbf{\%}$ & $\mathbf{N}$ & $\mathbf{\%}$ \\
\hline Corrected & 3 & 5.0 & 52 & 86.7 \\
\hline $\begin{array}{c}\text { Not } \\
\text { corrected }\end{array}$ & 57 & 95.0 & 8 & 13.3 \\
\hline Total & $\mathbf{6 0}$ & $\mathbf{1 0 0 . 0}$ & $\mathbf{6 0}$ & $\mathbf{1 0 0 . 0}$ \\
\hline
\end{tabular}

Table 5. Percentage of patients showing post transfusion PT correction in both major and mild coagulopathy

Chi square $=80.593, \mathrm{P}<0.001$.

The table shows that $95 \%$ of mild cases did not show any change in the post transfusion PT values where as $86.7 \%$ of major coagulopathy showed a significant improvement in post transfusion PT values.

\begin{tabular}{|c|c|c|}
\hline \multirow{2}{*}{ PT/INR } & \multicolumn{2}{|c|}{ Mild prolongation } \\
\hline & PT pre & PT post \\
\hline $\mathrm{N}$ & 60.0 & 60.0 \\
\hline Mean & 15.7 & 15.6 \\
\hline sd & 0.7 & 1.1 \\
\hline Minimum & 14.0 & 12.0 \\
\hline Q1 & 15.0 & 15.0 \\
\hline Median & 16.0 & 16.0 \\
\hline Q3 & 16.5 & 16.5 \\
\hline Maximum & 17.1 & 17.0 \\
\hline
\end{tabular}

Paired t test $\mathrm{t}=0.5941, \mathrm{p}=0.55, \mathrm{p}$ value shows it is not statistically significant.

\begin{tabular}{|c|c|c|}
\hline \multirow{2}{*}{ PT } & \multicolumn{2}{|c|}{ Major Prolongation } \\
\cline { 2 - 3 } & PT-pre & PT-post \\
\hline $\mathrm{N}$ & 60.0 & 60.0 \\
\hline Mean & 36.9 & 17.9 \\
\hline sd & 16.7 & 4.1 \\
\hline Minimum & 18.5 & 14.0 \\
\hline Q1 & 22.0 & 15.0 \\
\hline Median & 31.0 & 16.0 \\
\hline Q3 & 60.0 & 20.0 \\
\hline Maximum & 60.0 & 30.0 \\
\hline
\end{tabular}

Table 7. Post transfusion p value of major coagulopathy.

Paired $t$ test, $t=8.55, p<0.001, P$ value shows that it is significant.

\section{DISCUSSION}

Blood components have been in use since many years. Unlike red cell transfusion, where the traditional threshold of 10 $\mathrm{g} / \mathrm{dl}$ has been found to be unnecessarily high in some settings like surgery and intensive care by prospective randomized studies, such studies do not exist for FFP. ${ }^{9}$

FFP transfusion is appropriate in bleeding patients; patients undergoing invasive procedures with coagulopathy resulting from DIC, massive blood transfusion or liver failure and plasma exchange for thrombotic thrombocytopenic purpura.10-12 FFP is considered appropriate in patients with excessive warfarin effect only if they have a massive bleeding or are undergoing emergency surgery. In massive transfusion, there is no evidence that prophylactic replacement of FFP prevents the onset of abnormal bleeding or reduces transfusion requirements. Lastly, there are situations in which FFP is clearly not indicated like volume resuscitation, nutritional support in protein losing states like burns and plasma exchange procedures for conditions other than TTP. 12,13

In our study we followed up all the FFP requisition forms from patients with mild and major coagulopathies and their pre and post transfusion PT INR values were recorded. Out of the 120 requisition forms received, 60 cases were mild coagulopathy and 60 were of major.

According to our study in mild coagulopathy, all transfusions were prophylactic against bleeding, whereas in major coagulopathy $51.7 \%$ required therapeutic FFP transfusions.

The average amount of FFP given was 2 - 4 units, in both major and mild coagulation abnormality cases and median number was 2 which were similar to various studies. Our data suggest that under circumstances where FFP has a higher probability of patient benefit, namely, bleeding in a patient with INR prolongation, inadequate doses are currently administered to reliably correct coagulation factor deficiencies. This may explain, in part, the small decreases in INR prolongation that we observed after FFP transfusion, especially when the INR was only modestly prolonged. The reasons for transfusion of inadequate volumes of FFP are unclear and require further investigation.

One of the first studies to examine the effect of large volume FFP transfusions in correcting INR was in 1966 by Spector et al. thirteen patients with liver disease were given 
3-9 units FFP. The PT in only eight patients decreased to within $60 \%$ of normal activity. This effect was short-lived as elevation of coagulation factors declined by $50 \%$ within the first 2-4 hours after transfusion ${ }^{14}$. Forty years later at Massachusetts General Hospital, a study examined a larger cohort of 121 patients. The patients had an INR between 1.11.85 and a repeat INR within 8 hours after receiving FFP. The main indications for transfusion were pre-procedure with elevated INR and bleeding with elevated INR. Results showed that normalization of INR (INR $<1.1$ ) occurred in only $0.8 \%$ of patients and halfway to normalization of INR occurred in only $15 \%$ of patients 15 . On average, INR decreased by only 0.07 . Another study by Holland and Brooks which was a retrospective study showed a linear relationship to predict the change in INR per unit FFP. INR change $=0.37$ [pretransfusion INR] - 0.47.16 While pre-transfusion INR was predictive of the response to FFP, the strongest response was found when pre-transfusion INR was greater than 2 and not for mild elevations in INR. FFP treatment was minimally effective in correcting mild elevations in INR $<1.7$. Shinagare et al. found $64.9 \%$ improvement in the INR per unit of FFP and the improvement in the INR was more in those patients who had a high pretransfusion INR and improvement in the INR was less likely in patients who had a low pretransfusion INR. ${ }^{17}$

The above studies support the modification in recent guidelines indicating FFP transfusion as prophylaxis in nonbleeding patients with hereditary coagulation defects prior to invasive procedures. Coagulation activity is determined by ordering coagulation factor levels, such as factor $\mathrm{V}$ and factor VII, which are assayed from the PT. ${ }^{18}$ In general, prophylaxis may be indicated if the coagulation factor activity is $<30 \%$ or in patients with a history of recurrent significant bleeding with a coagulation factor activity $>30 \% .^{19}$

In a retrospective study of 115 patients, 44 critically-ill, non-bleeding patients received FFP. Only 36\% of this group had an INR "corrected" or reduced to $<1.5$ and received approximately $12 \mathrm{ml} / \mathrm{kg}$ of FFP. 71 patients did not receive FFP. Results showed no statistically significant decrease in INR or bleeding episodes, hospital deaths or length of stay in ICU. 20

In our study we examined the effect of FFP transfusion on PT INR values in 60 patients with mild and 60 patients with major coagulation abnormalities. We found that, in major coagulopathy, $86.7 \%$ patients showed significant improvement in PT INR (52/60), whereas in mild coagulopathy $95 \%$ cases $(57 / 60)$ did not show any improvement in PT INR values post transfusion. On statistical analysis, improvement in PT INR value after FFP transfusion in major coagulopathy showed significant $\mathrm{p}$ value $(\mathrm{P}<.001)$, however in patients with mild coagulopathy, FFP transfusion did not show any significant change $(\mathrm{p}=0.55)$

Despite commonly held views, it was found that mild-tomoderate elevation of the PT was not "corrected" by FFP transfusion in most of the patients. Only a small number of mild coagulation abnormality patients achieved normalization of PT following transfusion (5\%).

Our study differs from prior reports as we included a greater number of patients with a wide range of medical and surgical conditions, and we restricted our analysis to patients with a PT of fewer than 17 seconds (INR < 1.85) and compared with patients with PT greater than 18 and INR $>2$.
Multiple observational studies point to a lack of predictive effects for bleeding risk in patients with mild or moderate abnormalities determined in coagulation tests and, thus, indicates that coagulation results are only crude predictors of surgical bleeding. Our results provide a further basis to try to understand the manner in which physicians request blood transfusions and, thus, collect further evidence on the efficacy of FFP use.

Transfusion of a greater number of units of FFP might be expected to have a greater effect on correcting PT values after transfusion. However, there is little doubt that FFP transfusion can be effective at correcting laboratory abnormalities under the right circumstances.

In our study, $56.7 \%$ had packed red cell transfusions as most of them presented with bleeding whereas in mild, 43.3 $\%$ received packed red cell transfusion due to anaemia. In a study by Abdel Wahab, they estimated net RBC loss occurring around the time of FFP transfusion. They estimated the range of FFP transfused and found out to be $0-24$ units. Considering platelet transfusions, $11.7 \%$ of mild coagulopathy had platelet transfusions along with FFP whereas in major, 16.6 $\%$ had platelet transfusions. In mild coagulation abnormality we noted that even though the patients were not having significant thrombocytopenia or bleeding, platelet transfusions were given which might have been avoided. In major coagulopathy, patients who received platelet transfusion showed thrombocytopenia with bleeding. Here platelet transfusion is justifiable.

Conversely, the large number of FFP transfusions administered to non-bleeding patients without INR prolongation or with minor derangements suggests the possibility of widespread and inconsistent use of FFP without strong clinical rationale. Widespread use of FFP in nonbleeding patients, needs careful scrutiny. Most of the published studies reported that even in the presence of significant coagulopathy, FFP does not reduce the risk of bleeding. Our study revealed that most of the clinicians and surgeons in our hospital had the practice of using FFP as a prophylactic measure to prevent bleeding after major surgery and invasive procedures. FFP transfusion in chronic liver disease and coagulopathy with no bleeding also reflects the prophylactic treatment attitude of the clinicians in our institute.

Stronger evidence is needed to guide clinicians. Specifically, evidence suggesting that correction of INR prolongation is not required in the prophylactic clinical situation, could significantly alter current practice and potentially reduce demand for FFP. Continuing reports of an association between FFP administration and adverse patient outcomes, including the critically ill, further emphasize the requirement to improve the evidence base, especially for transfusion of FFP to nonbleeding patients.

Our study had limitations. We reported findings on a heterogeneous group of patients and could not completely eliminate the possibility that FFP would have a more substantial effect on the PT value for specific patient subgroups, although none were seen in our data. Furthermore, some of our patients received concurrent therapies, such as intravenous fluid therapy, at the time of the FFP transfusion that would have affected the post-FFP PT value. Another limitation of our study is that patients with a pretransfusion PT between 13.1 and 17 seconds (INR, 1.1 - 
1.5) who did not receive FFP were not studied. Therefore, no comparison can be made regarding the extent of blood loss and PT INR correction with and without FFP transfusion for patients with mild elevations in PT. The effect of FFP transfusion on the PT of healthy, non-bleeding volunteers has been previously studied.

This study highlights the pitfalls in use of fresh frozen plasma among clinicians. The high rates of inappropriate transfusion reflect the lack of knowledge as well as nonadherence to the guidelines among clinicians and about the appropriate laboratory criteria as the basis for FFP usage for clotting support.

Therefore, it seems that transfusion of FFP to patients with mild prolongation of the PT/INR may not be efficacious for correcting these laboratory abnormalities than usual medical care alone. Therefore, attempting to correct laboratory abnormalities in patients not meeting current FFP guidelines may expose patients to unnecessary infectious and non-infectious risks with no demonstrable benefit. The appropriate use of FFP requires an understanding of the properties of FFP and its inadequacies, as well as an appreciation of the complications of FFP usage. Given the absence of evidence of benefit coupled with the known risks of transfusion of FFP, FFP transfusion to non-bleeding patients in response to a mild to moderately prolonged PT value cannot be supported. Of note, the incidence of transfusion reactions observed in this study was consistent with the known incidence of transfusion complications reported elsewhere.

All these observations may help us to refine the institutional policies regarding usage of FFP. Since FFP is a precious product, we propose a preferential use of FFP for those patients who fulfil the guidelines and have a high pretransfusion PT INR.

\section{CONCLUSION}

After evaluating the usages of FFP, it was found that there is a generalized and widespread irrational use of FFP among specialists. To reduce the inappropriate usage of FFP the following strategies may be used-

1. The hospital transfusion guidelines should be established based on existing international guidelines.

2. Awareness programs for the clinicians should be conducted regularly.

3. In the requisition forms the appropriate indication for FFP transfusion should be mentioned.

4. Regular evaluation may help to reduce inappropriate use and plays a vital role in overseeing transfusion practices to ensure optimal use of blood and component therapy.

\section{REFERENCES}

[1] Nagareka K. Evaluation of fresh frozen plasma usage at a medical college hospital-a two year study. International Journal of Blood Transfusion and Immunohematology 2012;2:16-20.

[2] Bjerrum OJ, Jersild C. Class specific anti-IgA associated with severe anaphylactic transfusion reactions in a patient with pernicious anemia. Vox Sang 1971;21(5):411-24.
[3] Practice parameter for the use of fresh frozen plasma, cryoprecipitate and platelets. Fresh Frozen Plasma, Cryoprecipitate and Platelets Administration Practice Guidelines Development Task Force of the College of American Pathologists. JAMA 1994;271(10):777-81.

[4] Contreras M, Ala FA, Greaves M, et al. Guidelines for the use of fresh frozen plasma. British Committee for Standards in Hematology, Working Party of the Blood Transfusion Task Force. Transfus Med 1992;2(1):5763.

[5] National Health and Medical Research Council. Clinical practice guidelines on the use of blood components (red blood cells, platelets, fresh frozen plasma, cryoprecipitate) 2001. http://www.nhmrc.gov.au/publications/_files/cp78. pdf. Accessed November 1, 2005.

[6] Shariff MM, Maqbool S, Butt TK, et al. Justifying the clinical use of fresh frozen plasma - an audit. J Coll Physicians Surg Pak 2007;17(4):207-10.

[7] Palo R, Capraro L, Hovilehto S, et al. Population based audit of fresh-frozen plasma transfusion practices. Transfusion 2006;46(11):1921-5.

[8] Yeh CJ, Wu CF, Hsu WT, et al. Transfusion audit of fresh-frozen plasma in southern Taiwan. Vox Sang 2006;91(3):270-4.

[9] Chaudhary R, Sigh H, Verma A, et al. Evaluation of fresh frozen plasma usage at tertiary care hospital in North India. ANZ J Surg 2005;75(7):573-6.

[10] Prathibha R, Jayaranee S, Ramesh JC, et al. An audit of fresh frozen plasma usage in a tertiary referral centre in a developing country. Malays J Pathol 2001;23(1):41-6.

[11] Carson JL, Terrin ML, Barton FB, et al. A pilot randomized trial comparing symptomatic vs. haemoglobin-level-driven red blood cell transfusions following hip fracture. Transfusion 1998;38(6):522-9.

[12] Bracey AW, Radovancevic R, Riggs SA, et al. Lowering the hemoglobin threshold for transfusion in coronary artery bypass procedures: effect on patient outcome. Transfusion 1999;39(10):1070-7.

[13] Shanberge JN. Reduction of fresh frozen plasma usage through a daily survey and education program. Transfusion 1987;27(3):226-7.

[14] Spector I, Corn M, Ticktin HE. Effect of plasma transfusions on the prothrombin time and clotting factors in liver disease. New England Journal of Medicine 1966;275:1032-7. http://www.nejm.org/doi/full/10.1056/NEJM196611 102751902

[15] Abdel-Wahab OI, Healy B, Dzik WH. Effect of freshfrozen plasma transfusion on prothrombin time and bleeding in patients with mild coagulation abnormalities. Transfusion 2006;46(8):1279-85. http://www.ncbi.nlm.nih.gov/pubmed/16934060

[16] Holland LL and Brooks JP. Toward rational fresh frozen plasma transfusion: the effect of plasma transfusion on coagulation test results. American Journal of Clinical Pathology 2006;126(1):133-9. http://ajcp.ascpjournals.org/content/126/1/133.abst ract14. 
[17] Shinagare SA, Angarkar NN, Desai SR, et al. An audit of fresh frozen plasma usage and effect of fresh frozen plasma on the pre-transfusion international normalized ratio. Asian J Transfus Sci 2010;4(2):12832.

[18] West KL, Adamson C, Hoffman M. Prophylactic correction of the international normalized ratio in neurosurgery: a brief review of a brief literature. Journal of Neurosurgery 2011;114(1):9-18.
[19] Indications - New York State Council on Human Blood and Transfusion $\quad 2010$. www.wadsworth.org/labcert/blood_tissue/pdf/txopt salts0411.pdf. Accessed September 28, 2012.

[20] Dara SI, Rana R, Afessa B, et al. Fresh frozen plasma transfusion in critically ill medical patients with coagulopathy. Critical Care Medicine 2005;33(11):2667-71. 